\title{
Methodologies for Geotagging in FAT and ExFAT File Systems for Smart Phones
}

\author{
Keshava Munegowda \\ Principal Software Engineer, \\ Advanced Storage Division, \\ EMC Corporation \\ Bangalore, India
}

\author{
G T Raju, Ph. D \\ Professor and Head, \\ Department of computer Science \\ and Engineering, RNSIT, \\ Bangalore, India
}

\author{
Veeramanikandan Raju \\ Senior Member Technical Staff, \\ Texas Instruments \\ Bangalore, India
}

\begin{abstract}
File Allocation Table (FAT)file system is the most common file system used in embedded devices such as smart phones, digital cameras, smart TVs, tablets, etc. Typically these embedded devices use Solid State Drives (SSD) as storage devices. The ExFAT file system is future file system for embedded devices and it is optimal for SSDs. This paper discourses the methodologies for Geotagging as a file system metadata instead of file data in FAT and ExFAT file systems. The designed methodologies of this paper adheresthe compatibility with the FAT file system specification and existing ExFAT file system implementations.
\end{abstract}

\section{Keywords}

Cluster, Contiguous, ExFAT, EXIF, FAT, File system, Flash memories, GPS, MMC, Multimedia, Micro SD, NAND, NOR, SSD,Storage, Video, XMP.

\section{INTRODUCTION}

The Multimedia Cards (MMC) [1]/ Secure Digital (SD) [2] / Micro SD cards are composed of NOR and/or NAND flash memories [3]. The NOR and NAND flash memories are also called as "Solid State Drives". The Flash memories are default choice of any embedded device as they are low-priced, smaller size and higher storage capacity. The FAT [4] file system is the widely adopted file system in SSDs. The multimedia applications such as video imaging, audio/video playback and recording uses FAT file system for storing and retrieving the data of the multimedia files. The first version of FAT file system was FAT12 by Microsoft Corporation, later it was extended as FAT16 and further as FAT32 to support higher storage capacity. The FAT file system was initially developed to use on floppy disks and Hard Disk Drive (HDD) s. Since most of the operating systems of Personal Computer (PC) s such as Windows, Linux, Mac OS, etc. implement the FAT file system, this file system has become a default and world-wide compatible storage format for embedded devices. Usually the device with the implementation of FAT is recognized as removable storage media in a PC. Even though FAT file system does not define flash management techniques such as Wear-Levelling and Bad Block management [4], the embedded devices implements this file system along with the dedicated flash block management algorithms.

In FAT File system, the storage device is represented as group of linear clusters. A cluster is basic unit of data storage in FAT file system and it is a group of blocks or sectors of storage device. In FAT file system, the file or directory is a linked list of the clusters. The File Allocation Table stores the status of all available clusters of the device; Status of a cluster can be either allocated as part of file/directory data or free or reserved. An every entry of File Allocation Table indicates the status of corresponding cluster. The FAT file system specification limits the maximum supported storage size to $32 \mathrm{~GB}$. But, the maximum size supported by FAT32 implementation is $128 \mathrm{~GB}$. But, today the flash storage cards of more than $32 \mathrm{~GB}$ are available in market. The ExFAT [5] [6] file system is developed, by Microsoft Corporation, as successor of FAT32 file system. This file system is optimally designed to support large size flash storage cards with higher read and write performance. The maximum storage size supported by ExFAT file system in 128 peta bytes (PB).

Global PositioningSystem (GPS) based positioning [7][8]is the positioning technique commonly used inSmartphones. Most of Smartphone contains the GPS receiver which continuously receives the signals from the satellites. These satellite signals contain the exacttime of the message sent, and the location information. The GPS receiver usesthe received signals of four or more satellites to determine the current position based on trilateration.Many smart phones uses the multiple sensors improve the accuracy of the GPS position [9]. In Android based mobile phones, Location Manger [10] can be used for the Geotagging of multimedia files.

The eXtensible Metadata Platform (XMP) [11] and Exchangeable image file format (EXIF) [12] are the formats used to store the geographical coordinates and other related information in multimedia and Portable Document Format (PDF) files. In both XMP and EXIF formats, the Geographical data is always placed as multimedia file data. Due to this, file need to be opened and read operation is required to retrieve the geographical location of the file. This paper designs the techniques to place the geographical data of the file as attribute of the file instead of user data of the file. In FAT and ExFAT file systems, the attribute of the file contains the file name, size in bytes, date and time of creation and last write. The attribute of the file is referred as Meta data of the file. This paper includes the geographical data to the attribute of the file/directory by following methodologies.

i) Geotagging with Reserved Sectors of FAT and ExFAT file systems.

ii) Geotagging with Reserved Clusters of FAT file systems.

The files created/updated with above techniques adheres the compatibility with the existing FAT and ExFAT file system implementations and specifications. This means, files created with Geographical data as attributes are accessible for read and write operations in other FAT and ExFAT file system implementations without Geotagging facility. The Geotagging methodologies described in this paper stores the geographical 
data of all data clusters of the FAT and ExFAT file system in "Geo-Attribute Table". This Geo-Attribute Table can be placed in either in reserved sectors or in clusters which are marked as reserved and not allocated to any file.

\section{GEOTAGGING WITH RESERVED SECTORS OF FAT AND EXFAT FILE SYSTEM}
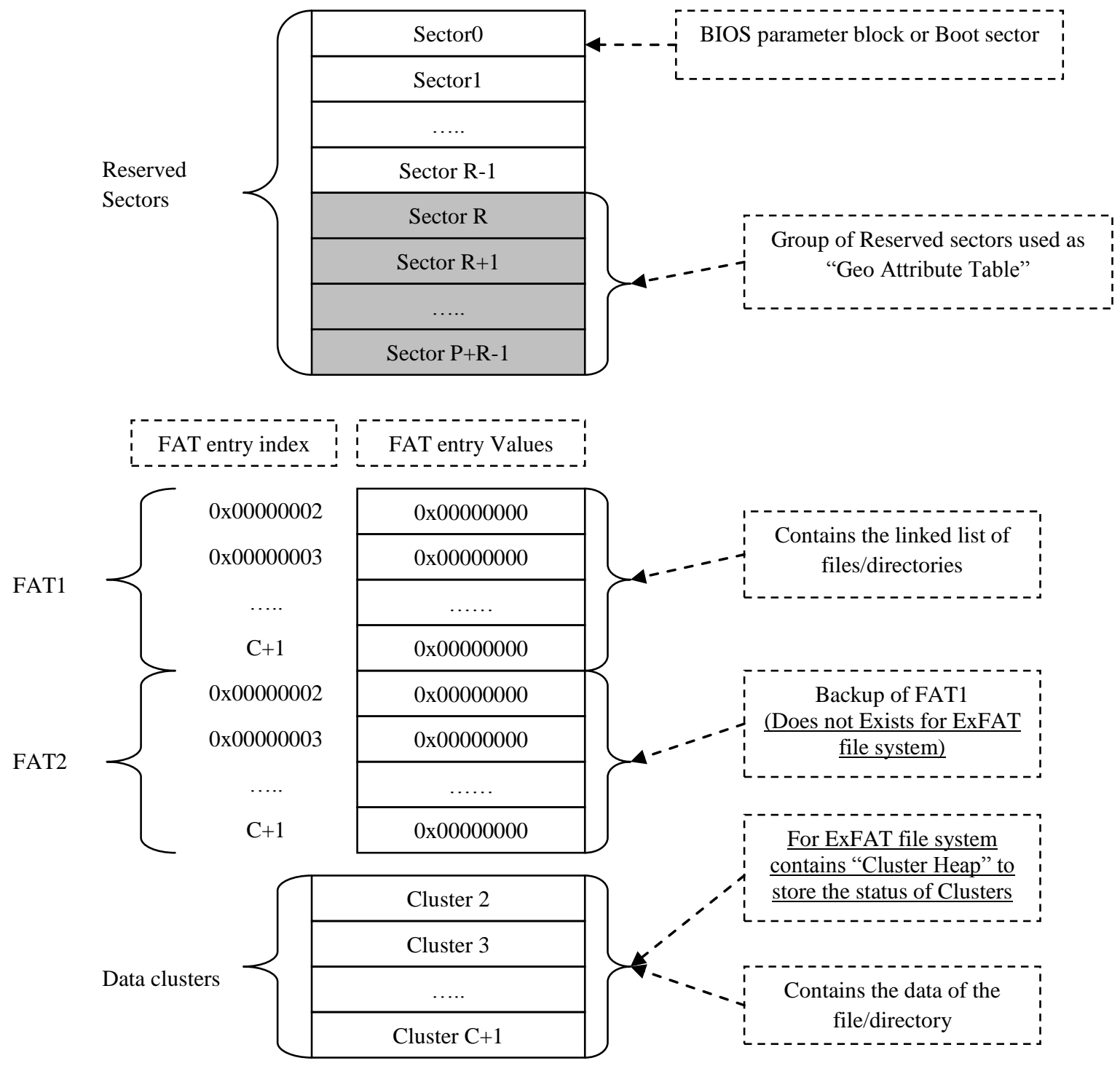

Fig 1: Organizations of FAT and ExFAT file systems with reserved sectors

The FAT file system requires at least one sector as reserved sector as the BIOS parameter block or boot sector. This boot sector contains the information about the partition of the storage device. If the FAT file system accommodates more sectors as reserved sectors, then except the first sector other sectors can be used to store the geographical information of the files/directories.
Figure 1 shows the FAT file system organization with first reserved sector as boot sector and several more reserved sectors between boot sector and File Allocation Table. The Reserved sectors, FAT1, FAT2 and data clusters exist in logically linear fashion in FAT file system. The Reserved sectors of the file system are hidden from the user and hence it avoids the accidental writes to these sectors. The reserved sectors are never allocated to any file/directory.

Typically, the longitude and latitude forms the basic geographical information. Longitude can be stored as 4 bytes value and also latitude can be as 4 bytes value, thus minimal 8 bytes are required for Meta data of the file. These reserved 
sectors are hidden from the user and hence prevents the user updating the geographical attributes of the file. Table 1 shows the symbols/parameters used to derive the formula for determine the number of reserved sectors for Attribute Table.

\begin{tabular}{|c|c|}
\hline Parameter & Description \\
\hline A & $\begin{array}{l}\text { Total Number of sectors in a partition of the } \\
\text { storage device }\end{array}$ \\
\hline B & Logical/physical block size in Bytes \\
\hline $\mathrm{C}$ & Number of clusters \\
\hline $\mathrm{E}$ & $\begin{array}{l}\text { FAT entries size in Bytes; E will be } 3 / 2 \text { in } \\
\text { case of FAT12, } 2 \text { in case of FAT } 16 \text { and } 4 \text { in } \\
\text { case of FAT32 file systems. }\end{array}$ \\
\hline $\mathrm{F}$ & $\begin{array}{l}\text { Number of File Allocation Tables; Usually } \\
\text { this value will be } 2\end{array}$ \\
\hline G & Sectors per File Allocation Table. \\
\hline $\mathrm{K}$ & $\begin{array}{l}\text { Entry size in Attribute Table; Typically K } \\
\text { will be } 8 \text { bytes. The first } 4 \text { bytes are used to } \\
\text { store the longitude and remaining } 4 \text { bytes are } \\
\text { used to store longitude. }\end{array}$ \\
\hline $\mathrm{R}$ & $\begin{array}{l}\text { Number of Reserved Sectors. This value } \\
\text { should be at least } 1 \text { to indicate the BPB } \\
\text { (BIOS parameter block) or Boot sector }\end{array}$ \\
\hline $\mathrm{S}$ & $\begin{array}{l}\text { Number of Sectors per cluster; FAT32 } \\
\text { specification defines the this value } \\
\text { depending on value of A. }\end{array}$ \\
\hline $\mathrm{T}$ & $\begin{array}{l}\text { Number of Geographical entries per cluster. } \\
\text { Each entry is of K bytes }\end{array}$ \\
\hline $\mathrm{N}$ & Number of Clusters per Attribute Table \\
\hline $\mathrm{P}$ & Number of Sectors per Attribute Table \\
\hline
\end{tabular}

Note that, the parameters A and B can be retrieved from the storage device driver and value for parameters $\mathrm{E}, \mathrm{F}$ and $\mathrm{S}$ are defined by the FAT and ExFAT file system specifications. The values for the parameters $\mathrm{K}, \mathrm{R}$ can be user configured. While formatting the storage device the calculation of parameters $\mathrm{G}, \mathrm{P}$ and $\mathrm{C}$ are as follows.

The Parameter A (Total number of sectors in device) is

$$
A \equiv R+F \times G+C \times S
$$

The above equation (1) has two unknown variables $\mathrm{G}$ and $\mathrm{C}$.

Numberofclusters $(C) \equiv$ NumberofEntrie $\sin F A T$ $C \equiv G \times$ NumberofEntriespersS ector

$$
C \equiv G \times(B / E)
$$

Combining above equations (1) and (2)

$$
\begin{aligned}
& A \equiv R+F \times G+G \times(B / E) \times S \\
& A \times E \equiv R \times E+F \times G \times E+G \times B \times S \\
& A \times E \equiv R \times E+(F \times E+B \times S) \times G \\
& (A \times E)-(R \times E) \equiv(F \times E+B \times S) \times G \\
& \therefore G \equiv \frac{(E \times(A-R))}{((F \times E)+(B \times S))}
\end{aligned}
$$

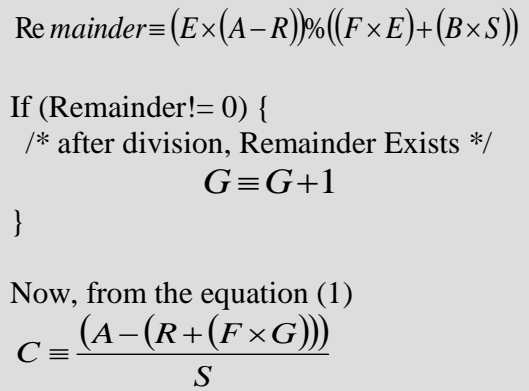

If the Geographical information is stored in the reserved sectors $(\mathrm{P})$, then, the calculation to determine the parameter A changes as follows:

$$
\begin{aligned}
& A \equiv R+F \times G+C \times S+P \\
& P \equiv\left(\frac{K}{E}\right) \times G
\end{aligned}
$$

For FAT32, $\mathrm{E}$ is 4 and $\mathrm{K}$ value is 8 ,

$$
\therefore P \equiv 2 \times G
$$

Now, combining equation (2), (6) and (7)

$$
A \equiv R+F \times G+G \times(B / E) \times S+(K / E) \times G
$$

Now, from equation (8) the parameter $\mathrm{G}$ is derived as

$$
\therefore G \equiv \frac{(E \times(A-R))}{((F \times E)+(B \times S)+K)}
$$

Re mainder $\equiv(E \times(A-R)) \%((F \times E)+(B \times S)+K)$

If (Remainder $!=0)\{$

/* after division, Remainder Exists */

$$
G \equiv G+1 \quad \text { (11) }
$$

\}

Now, from the equation (6), the parameter $\mathrm{C}$ is derived as

$$
C \equiv \frac{(A-(R+(F \times G)+P))}{S}
$$

In case of FAT file system, during the file write, the data clusters are allocated by writing the cluster entries of File allocation table as allocated. In case of ExFAT file system, during file write if the contiguous free clusters are available then those clusters are allocated by marking the cluster bit entries of Cluster heap [13][14] as allocated. But, for both the file system each cluster entry in Attribute table is always associated with 8 bytes (denoted as symbol $\mathrm{K}$ ) entry in the Attribute table. Each 8 bytes entry stores the longitude, latitude of physical location at which file data being written/updated. If $X$ is the first data cluster of the file, then the longitude of the file will be stored at the index $2 *(X-2)$ and the latitude value will be stored at the index $[2 *(\mathrm{X}-2)]+1$ of Geo-Attribute Table. 


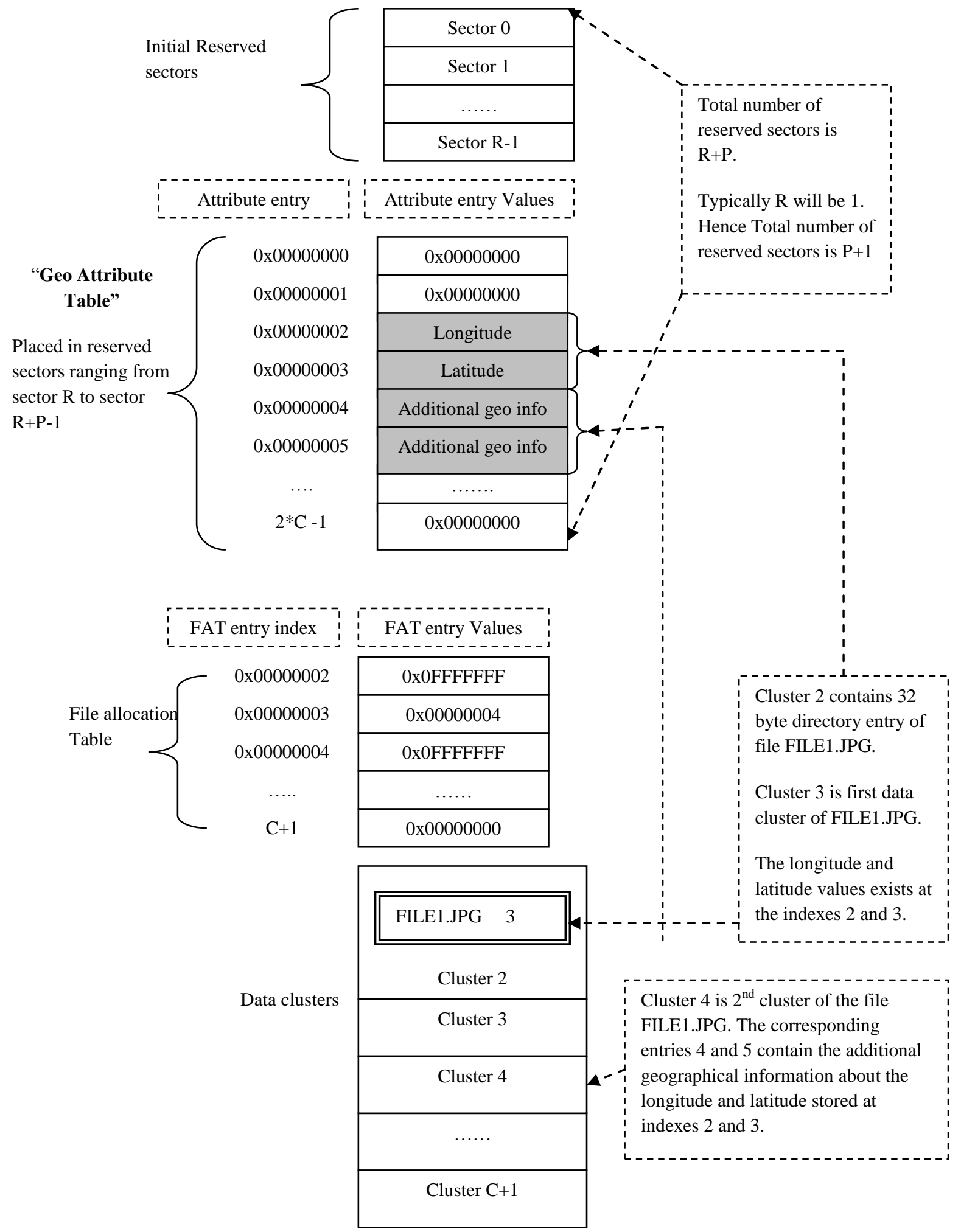

Fig.2. FILE1.JPG with geographical information in reserved sectors 
The organization of the file data with geographical information is shown in figure 2. Note that, cluster 2 is the first data cluster and it is the first data cluster of the root directory. For FILE1.JPG file the first data cluster is 3 . The longitude and latitude of this file are stored at the index 2 and 3 respectively. Additional geographical information about the longitude and latitude of the file can be stored in the indexes calculated for the $2^{\text {nd }}$ cluster onwards to a file. For example, if cluster 4 is the second data cluster of the file FILE1.JPG, the corresponding geographical entries 4 and 5 contains the additional information about the longitude and latitude of the file FILE1.JPG. Similarly if cluster 5 is $3^{\text {rd }}$ cluster of the file FILE1.JPG, then the corresponding entries 6 and 7 contains the additional geographical information about the file. The user can reserve the $\mathrm{R}+\mathrm{P}$ number of sectors as reserve sectors of file system during file system format operation. The file system format operation removes all content of the file system; hence, if the file system already has the data and user requires to geographical data for the new files created then geographical information can be stored in the reserved clusters of the file system.

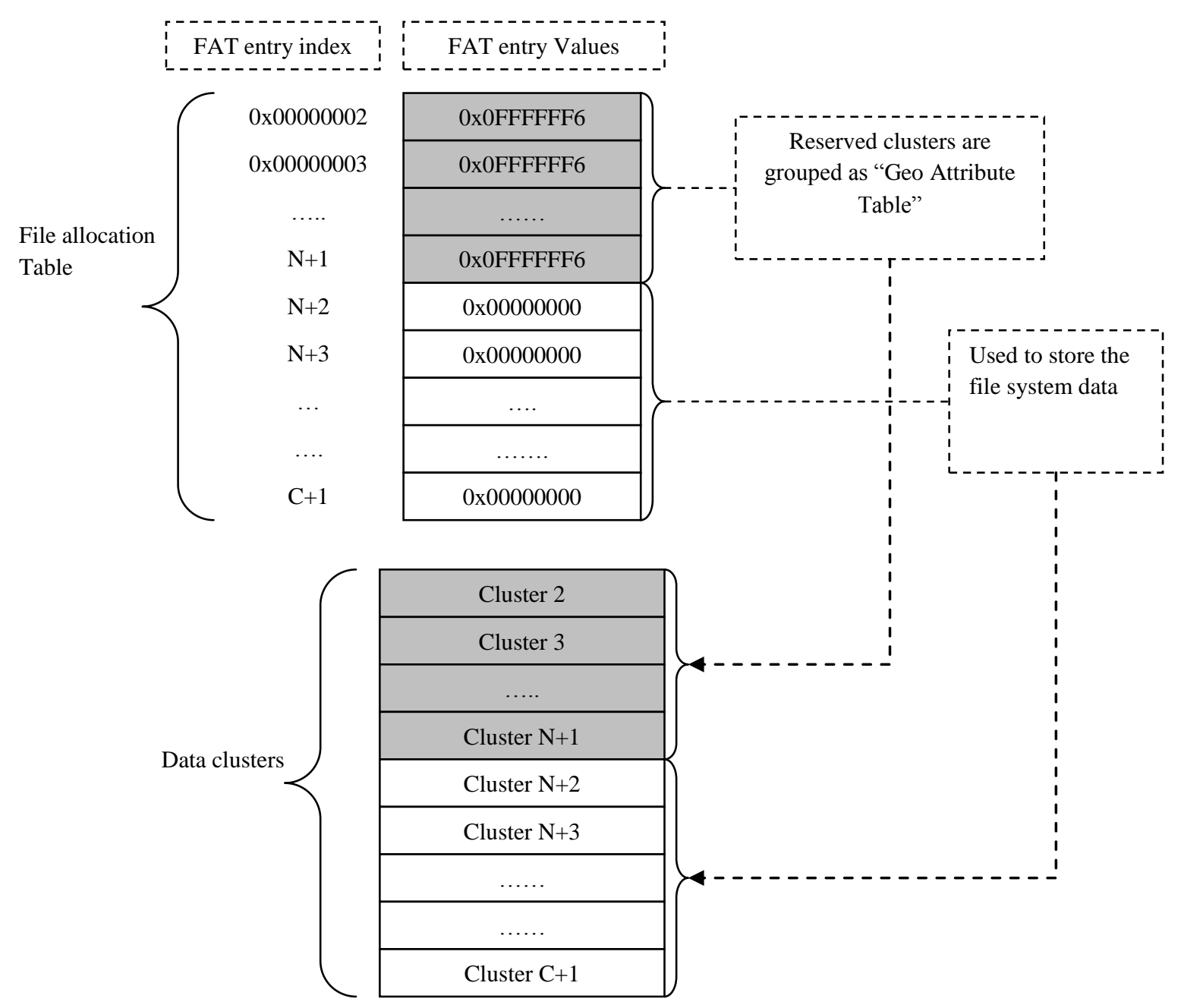

Fig.3. FAT file system with reserved clusters 


\section{GEOTAGGING WITH RESERVED CLUSTERS OF FAT FILE SYSTEM}

The Geo-Attribute Table can be placed in the reserved clusters also.These reserved clusters are hidden from the user and hence prevents the user updating the geographical attributes of the file. Figure 3 show that FAT file system with clusters numbered from 2 to $\mathrm{N}+1$ are marked as reserved.

If the Geographical information is stored in the reserved clusters, the number of geo graphical entries per cluster (T) is determined as follows:

$$
T \equiv \frac{(B \times S)}{K}
$$

The number of clusters (N) to be reserved as Geo Attribute Table for FAT file system is determined using following formula

$$
N \equiv \frac{C}{(T+1)}
$$

$\operatorname{Re} \min d e r \equiv C \%(T+1)$

If (Remainder! $=0)\{$

$I^{*}$ after the division operation, Remainder exists*/

$N \equiv N+1$

During the file write, the data clusters are allocated by marking the cluster entries of File allocation table as allocated. Each cluster entry in File allocation table is always associated with 8 bytes entry in the Attribute table. Each 8 bytes (denoted as symbol $\mathrm{K}$ ) entry stores the longitude, latitude of physical location at which file data being written/updated. If $\mathrm{N}$ is number of clusters reserved for GeoAttribute table and $\mathrm{X}$ is the first data cluster of the file, then the longitude of the file will be stored at the index $2 *(\mathrm{X}-\mathrm{N}-2)$ and the latitude value will be stored at the index $[2 *(\mathrm{X}-\mathrm{N}-$ $2)]+1$ of Geo-Attribute Table. The organization of the file data with geographical information is shown in figure 4. Note that, cluster numbered $\mathrm{N}+2$ is the first data cluster and it is the first data cluster of the root directory. For FILE1.JPG file the first data cluster is $\mathrm{N}+3$. The longitude and latitude of this file are stored at the index 2 and 3 respectively. Additional geographical information about the longitude and latitude of the file can be stored in the indexes calculated for the $2^{\text {nd }}$ cluster onwards to a file. For example, if cluster N+4 is the second data cluster of the file FILE1.JPG, the corresponding geographical entries 4 and 5 contains the additional information about the longitude and latitude of the file FILE1.JPG. Similarly if cluster N+5 is $3^{\text {rd }}$ cluster of the file FILE1.JPG, then the corresponding entries 6 and 7 contains the some more geographical information about the file.

The reserved clusters can exist only on FAT file system, in case of ExFAT file system, an hidden file with "N" number of clusters can created in the root directory to store the geographical information of every cluster of file system. The reserved cluster technique does not require a file system format the geotagging can performed without losing existing file system data. But, the reserved clusters technique depends on the availability of required number of free clusters to trigger the geotagging. If there are not enough free clusters in the file system, then directory compaction [15] can be executed to generate the free clusters from the clusters allocated to directories of file system.

\section{CONCLUSION}

The methodologies, geotagging with reserved sectors and reserved clusters of file system, discussed in this paper enhances the file attributes of the FAT and ExFAT file system by including the geographical information of files. The files created/updated with above techniques adheres the compatibility with the existing FAT and ExFAT file system implementations and specifications. This means, files created with Geographical data as attributes of files are accessible for read and write operations in other existing FAT and ExFAT file system implemented embedded devices. But, the existing FAT and EXFAT implementations may not extract the geographical information of files and also the reserved sectors and clusters will not be allocated to a file or directory while creation or data write operations. These techniques avoid the file open and data read operations to retrieve the geographical data of the multimedia files. The Table 1 summarizes these differentiating features.

Table 1. Differences between Geotagging in reserved

\begin{tabular}{|c|c|c|c|}
\hline Feature & $\begin{array}{c}\text { Geo- } \\
\text { Attribute in } \\
\text { Reserved } \\
\text { Sectors }\end{array}$ & $\begin{array}{c}\text { Geo- } \\
\text { Attribute in } \\
\text { Reserved } \\
\text { Clusters } \\
\end{array}$ & $\begin{array}{l}\text { XMP and } \\
\text { EXIF } \\
\text { formats }\end{array}$ \\
\hline $\begin{array}{c}\text { Location of } \\
\text { Geographical } \\
\text { information }\end{array}$ & $\begin{array}{c}\text { File Meta } \\
\text { data }\end{array}$ & $\begin{array}{c}\text { File Meta } \\
\text { Data }\end{array}$ & $\begin{array}{l}\text { File user } \\
\text { data }\end{array}$ \\
\hline Security & $\begin{array}{c}\text { Secure } \\
\text { because } \\
\text { reserved } \\
\text { sectors are } \\
\text { hidden from } \\
\text { user }\end{array}$ & $\begin{array}{c}\text { Secure } \\
\text { because } \\
\text { reserved } \\
\text { clusters are } \\
\text { hidden from } \\
\text { user }\end{array}$ & No security \\
\hline $\begin{array}{c}\text { Retrieval of } \\
\text { Geographical } \\
\text { information }\end{array}$ & $\begin{array}{c}\text { File open } \\
\text { operation is } \\
\text { not required }\end{array}$ & $\begin{array}{c}\text { File open } \\
\text { operation is } \\
\text { not required }\end{array}$ & $\begin{array}{l}\text { File open } \\
\text { operation is } \\
\text { required }\end{array}$ \\
\hline $\begin{array}{c}\text { Compatibility } \\
\text { with Exiting } \\
\text { FAT and } \\
\text { ExFAT } \\
\text { implementation }\end{array}$ & Yes & Yes & $\begin{array}{c}\text { Not } \\
\text { Applicable }\end{array}$ \\
\hline $\begin{array}{l}\text { Dynamic } \\
\text { insertion and } \\
\text { removal of } \\
\text { Geotagging }\end{array}$ & No & Yes & Yes \\
\hline File type & $\begin{array}{l}\text { Applicable } \\
\text { for Any } \\
\text { type of files; } \\
\text { Applicable } \\
\text { for text, } \\
\text { binary and } \\
\text { multimedia } \\
\text { files }\end{array}$ & $\begin{array}{l}\text { Applicable } \\
\text { for Any } \\
\text { type of files; } \\
\text { Applicable } \\
\text { for text, } \\
\text { binary and } \\
\text { multimedia } \\
\text { files }\end{array}$ & $\begin{array}{l}\text { Applicable } \\
\text { only for } \\
\text { multimedia } \\
\text { files }\end{array}$ \\
\hline $\begin{array}{c}\text { Applicable File } \\
\text { systems }\end{array}$ & $\begin{array}{l}\text { Applicable } \\
\text { for FAT and } \\
\text { ExFAT file } \\
\text { systems }\end{array}$ & $\begin{array}{l}\text { Applicable } \\
\text { for FAT file } \\
\text { system }\end{array}$ & $\begin{array}{l}\text { Applicable } \\
\text { for Any/All } \\
\text { file systems }\end{array}$ \\
\hline
\end{tabular}
sectors, reserved clusters and XMP / EXIF formats 


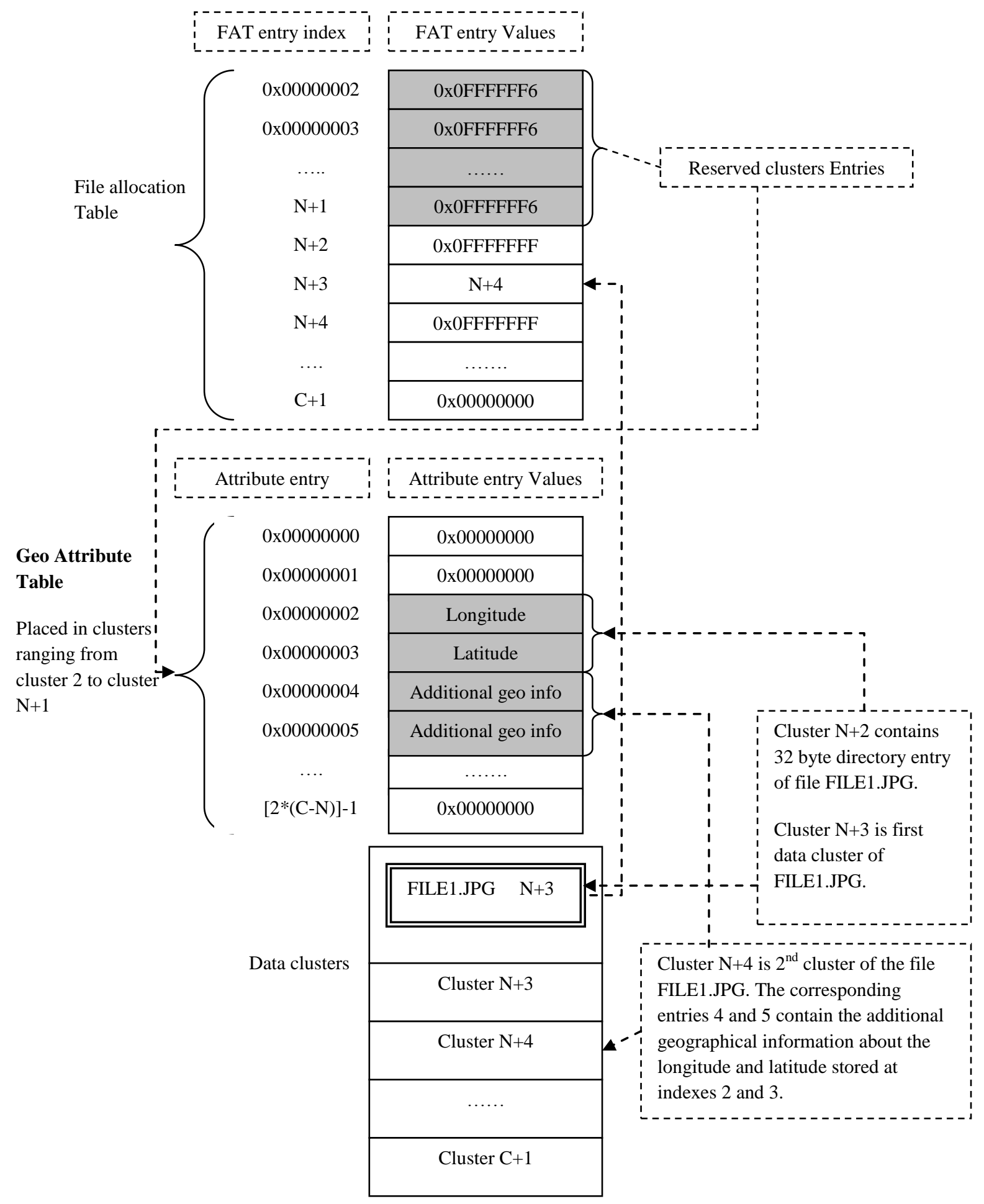

Fig 4: FILE1.JPG with geographical information in reserved clusters 


\section{REFERENCES}

[1] MMC 4.4 standard, JEDEC, 2008.

[2] SD Specifications Part 1: Physical Layer Simplified Specification version 4.10, SD card Association, January $22,2013$.

[3] Supriya Kulkarni P, Jisha P, "Study of Bad block management and Wear leveling in NAND flash memories", International Journal of Research in Engineering and Technology (IJRET), Volume 2, Issue 10, October 2013

[4] FAT32 File System Specification, FAT: General Overview of On-Disk Format, Microsoft, 2000.

[5] Ravishankar V.Puipeddi, Vishal V.Ghotge, Ravinder S.Thind, "Quick file name look up using name hash", USPTO Patent: 8321439, granted on November 27, 2012.

[6] Keshava Munegowda, Venkatraman S, Dr. G T Raju, "The Extend FAT file system: Differentiating with FAT32 file system", Linux Conference, Prague, Czech Republic, Europe, October 2011.

[7] Global Positioning System standard positioning service signal specification, $2^{\text {nd }}$ Edition, June 1995.

[8] U.S. Government, "Official U.S. Government information about the Global Positioning System (GPS) and related topics. " Internet: www.gps.gov, Apr. 20, 2012.
[9] Soyoung Hwang, Donghui Yu, “ GPS Localization Improvement of Smartphones Using Built-in Sensors", International Journal of Smart Home, Volume 6, Number 2, July 2012.

[10] Isha Sahu, Ishita Chakrborty, "Understanding Location Manager in Android and Implementing an Optimal Image Geotagging Application”, International Journal of Computer Trends and Technology (IJCTT), volume 4 Issue 6, 2013.

[11] XMP Specification Part3, Storage in files, May 2013.

[12] Exchangeable Image file format for digital still cameras: Exif version 2.3, December 2012

[13] Ravishankar V.Puipeddi, Vishal V.Ghotge, Ravinder S.Thind, "Contiguous File Allocation in Extensible File system", USPTO Patent: 8,606,830, granted on November 20, 2013.

[14] Keshava Munegowda, Dr. G T Raju, Veera Manikandan Raju, "Cluster Allocation Strategies of the ExFAT and FAT File Systems: A Comparative Study in Embedded Storage Systems", Proceedings of International Conference on Advances in Computing , Springer,Volume 174, 2012.

[15] Keshava Munegowda, Dr. G T Raju, Veera Manikandan Raju, "Directory Compaction Techniques for Space Optimizations in ExFAT and FAT File Systems for Embedded Storage Devices", IJCSI International Journal of Computer Science Issues, Vol. 11, Issue 1, No 2, January 2014. 